\title{
Administración pública en el socialismo desde la perspectiva de Ludwig Von Mises
}

\author{
Borgucci, Emmanuel*
}

\section{Resumen}

En los últimos diez años en Venezuela se ha producido un fuerte cuestionamiento tanto a la empresa como a la gerencia privada y se ha presentado como alternativa el denominado "Socialismo del Siglo XXI". El objetivo de este artículo es exponer los principios que sustentan el modelo de gestión administrativa socialista desde la perspectiva de los economistas austriacos. En consecuencia, la metodología empleada fue documental o bibliográfica, mediante la revisión y análisis crítico de los aportes que Ludwig von Mises expuso en Bureaucracy de 1944, Omnipotent Government de 1944 y Human Action de 1949. Se concluye que el gerente socialista, afronta, en términos generales, las mismas disyuntivas que un gerente en la economía capitalista, pero se encuentra con menos herramientas para minimizar los efectos negativos de las decisiones surgidas en la planificación central. Esta limitación se encuentra en: la carencia de la herramienta del cálculo económico basado en el mercado; la separación entre fines en sí fijados por criterios políticos y medios; y la imposibilidad de ajustar sus expectativas a los cambios del entorno económico, debido a que no hay mercado o si existe no sirve de referente.

Palabras clave: Socialismo de Mercado, Socialismo del Siglo XXI, Burocracia, Intervencionismo, estatismo.

\section{Public Administration under Socialism from the Perspective of Ludwig Von Mises}

\begin{abstract}
The last ten years in Venezuela have produced an intense questioning of the corporation as well as of private management, and an alternative has been presented, called "Socialism of the XXIst Century." The objective of this article is to expound the principles that support the socialist administrative management model from the perspective of Austrian economists. Consequently, the methodology used was documentary or bibliographic, through a review and critical analysis of the contributions
\end{abstract}

\section{Recibido: 13-10-10. Aceptado: 27-09-11}

* Doctor en Ciencias Sociales, Universidad Central de Venezuela. Profesor de la Facultad de Ciencias Económicas y Sociales de la Universidad del Zulia. e-mail: eborgucci@yahoo.com 
explained by Ludwig von Mises in Bureaucracy (1944), Omnipotent Government (1944) and Human Action (1949). Conclusions are that the socialist manager faces, in general terms, the same junctures faced by a manager in the capitalist economy, but finds fewer tools to minimize the negative effects of decisions arisen in the central planning process. This limitation is found in: a lack of economic calculation tools based on the market; the separation between goals fixed by political and mediatic criteria; the impossibility of adjusting expectations to changes in the economic environment, since there is no market or, if it exists, it does not serve as a referent.

Keywords: Market socialism, socialism for the $21^{\text {th }}$ century, bureaucracy, Interventionism, statism.

\section{Introducción}

Durante los últimos diez años en Venezuela ha cobrado impulso la crítica al modelo capitalista de gerencia en vigencia tanto en las empresas de servicio público como en las empresas con fines de lucro. Estas críticas recorren todo un espectro de aspectos que van desde la tendencia al individualismo, el deseo primario por las ganancias, la conducta especulativa o la existencia de monopolios en el sector agroindustrial.

Frente a estos señalamientos, la solución que se plantea es el reforzamiento de la intervención del Estado en el proceso económico por medio de compra de empresas de servicio que estaban en manos privadas tales como la Compañía Anónima Nacional Teléfonos de Venezuela (CANTV), la Siderúrgica del Orinoco (SIDOR) nacionalizada el 9 de abril de 2008, la Electricidad de Caracas (ELECAR), la compra de Lácteos Andina, la recuperación del hato el Frío (operadora de una estación biológica en 63.000 hectáreas de tierras de los llanos con 25.000 cabezas de ganado), el control de cambios, el control de precios en bienes de la canasta básica, o la modificación de los acuerdos contratos de la denominada "Apertura Petrolera”, entre otras iniciativas.
Esta discusión plantea nuevamente en Venezuela el debate entre las bondades y defectos de los criterios capitalistas para poner en marcha organizaciones que realizan actividades económicas y las bondades y deficiencias del modelo de gestión socialista. Este viejo debate que surgió en los escritos de Oskar Lange, Abba Lerner, Ludwig von Mises, Peter Schumpeter (1994a) y Friedrich von Hayek en la década de los 20 y 30 del siglo XX cobra impulso en Venezuela entre los proponentes del denominado "Socialismo del Siglo XXI" y quienes defienden las ideas denominadas "neo-liberales".

Si bien es cierto que Oskar Lange ofreció pruebas acerca de la posibilidad en el socialismo de la asignación eficiente de recursos en su réplica a Friedrich von Hayek y Ludwig von Mises y que autores como Joseph Schumpeter consideraran que los problemas de socialismo se concentran en la existencia de grandes burocracias (Schumpeter, 1994b[1942]) o de "Nomenclaturas"1 (Voslensky, 1981), los sucesos de la Perestroika y la posterior desintegración de la principal "nomenklatura" burocrática del mundo llamada la Unión Soviética, dio un nuevo significado a lo que Ludwig von Mises expuso en $\mathrm{Bu}$ reaucracy de 1944, Omnipotent Government también de 1944, así como Hu- 
man Action de 1949. En tal sentido el objetivo de este artículo es exponer los fundamentos de la crítica de uno de los economistas austriacos más importantes que abordaron los problemas de la administración pública, al menos de una manera directa ${ }^{2}$.

\section{El socialismo de planificación centralizada y el socialismo de mercado}

El pensamiento socialista "Socialismo científico" ha recorrido un largo trecho de propuestas que partieron, desde el punto de vista económico, con la teoría del valor-cantidad-trabajo desarrollada por Marx (1976 [1867] a).

Desde el punto de vista de la teoría del valor, un bien contiene valor (verdadero precio) (Gregory y Stuart, 1989): "por ser encarnación o materialización del trabajo humano abstracto" (Marx, 1976 [1867]a: 6) y su magnitud se mide por la cantidad de trabajo socialmente ne- cesario: "o sea, el tiempo de trabajo socialmente necesario para su producción" (Marx, 1976 [1867]a: 7). Esto es, que la cantidad de trabajo se mide por el tiempo de su duración y éste en fracciones de tiempo. Esta magnitud permanecerá inalterable y cambia cuando cambia la capacidad productiva del trabajo (Marx, 1976 [1867] a: 7$)^{3}$. Por otra parte, como acota Schumpeter (1994a), Marx distinguió entre trabajo, cuya cantidad se mide en horas y "fuerza de trabajo" (Arbeitskraft) cuyo valor es igual a la cantidad de trabajo contenida en los bienes que le permite reproducir su "fuerza de trabajo".

Por lo tanto, el valor de una mercancía será igual al costo directo de trabajo (capital variable), los costos indirectos del trabajo (capital constante) y la plusvalía (ganancia para el capitalista). Pero ese producto que se transforma en mercancía requiere ser cambiada en el mercado por medio de dinero, tal y como Marx lo expresó en los Grundrisse:

1 Para Michael Voslensky la nomenclatura se puede definir de la siguiente manera: "Son las relaciones de gestión, tal como, en los países del socialismo real, es practicada por la omnipotente administración política; es decir; esencialmente por los comité políticos y los secretariados de los comités centrales de los partidos comunistas en el poder" (1981: 17-18).

2 Si bien es cierto que pudieron existir obras previas de este autor relacionadas con el socialismo y la gerencia y que se publicó un día después de Road to Serfdom de Friedrich von Hayek, obra que tuvo más impacto, según Nash (1987 [1976]), este y los demás libros de Mises ejercieron un fuerte impacto en lo que después se convertiría en el movimiento libertario estadounidense de defensa de las ideas de la libre empresa y la libertad económica frente al totalitarismo que en aquel momento era hegemónico en Europa.

3 Para Marx: "La capacidad productiva del trabajo depende de una serie de factores, entre los cuales se cuentan el grado medio de destreza del obrero, el nivel de progreso de la ciencia y de sus aplicaciones, la organización social del proceso de producción, el volumen y la eficacia de los medios de producción y las condiciones naturales" (1976[1867]: 7). Por lo tanto, agrega el autor: "La magnitud del valor de una mercancía cambia en razón directa a la cantidad y en razón inversa a la capacidad productiva del trabajo que en ella se invierte" (Marx, 1976[1867]a: 8). 
"The process, then, is simply this: The product becomes a commodity, i.e. a mere moment of exchange. The commodity is transformed into exchange value. In order to equate it with itself as an exchange value, it is exchanged for a symbol which represents it as exchange value as such. As such a symbolized exchange value, it can then in turn be exchanged in definite relations for every other commodity" (Marx, 2008 [1857-61]: 81).

Es interesante señalar que Marx en los Grundrisse, estableció como ley de la comunidad el garantizar, de manera planificada, una valoración y una distribución del trabajo-tiempo de la forma más equitativa posible para cumplir con las necesidades sociales. La siguiente cita es elocuente en este sentido:

"On the basis of communal production, the determination of time remains, of course, essential. The less time the society requires to produce wheat, cattle, the more time it wins for other production, material or mental. Just as in the case of an individual, the multiplicity of its development, its enjoyment and its activity depends on economization of time. Economy of time, to this all economy ultimately reduces itself. Society likewise has to distribute its time in a purposeful way, in order to achieve a production adequate to its overall needs; just as the individual has to distribute his time correctly in order to achieve knowledge in proper proportions or in order to satisfy the various demands on his activity. Thus, economy of time, along with the planned distribution of labour time among the various branches of production, remains the first econo- mic law on the basis of communal production (Marx, 2008 [1857-61]: 114).

La anterior cita es reflejo de la necesidad, por parte de quienes detentan el poder político, de establecer un sistema de planificación centralizada de las actividades económicas para establecer una distribución del tiempo entre las diferentes actividades económicas para cubrir tanto las necesidades sociales como las económicas. Para que lo anterior suceda, se debe consolidar el papel del proletariado como clase dominante (Lenin, 2007[1917]) y contemporáneamente despojar a la burguesía de los medios de producción y reorganizar la producción sobre la base de relaciones sociales socialistas. En conclusión: "En lugar del gobierno sobre las personas aparece la administración de las cosas y la dirección de procesos de producción. El Estado no "se suprime", sino que se extingue" (Lenin, 2007[1917]: 50).

Sin embargo, la aplicación directa de la teoría del valor de Marx presentó, al menos dos problemas serios: el de la asignación de recursos y de adecuar la planificación a la "ley del valor". De esta manera, los economistas soviéticos de la década de los años 20 del siglo XX se concentraron en diseñar "técnicas de planificación económica nacional” (Gregory y Stuart, 1989). Según Lange (1976) se dieron una serie de circunstancias que determinaron la necesidad, según él, de la planificación "socialista". En primer lugar, en el socialismo seguía operando la "ley del valor", por la coexistencia de diferentes tipos de propiedad. En segundo lugar: "El socialismo ocurrió primero en los países que el imperialismo había impedido desarrollarse 
según los lineamientos capitalistas tradicionales" (Lange, 1976: 13).

Esta planificación debió, según el autor mencionado, sustentarse en la "pequeña producción de mercancías" y de ahí la importancia de promoción de "cooperativas socialistas". Según Lange (1976), la planeación económica comienza con la intervención del Estado en la economía para liquidar las relaciones de producción capitalistas, el establecimiento de las relaciones socialistas de producción y el control de los sectores no socialistas. En palabras de Lange "La base que hace posible el control del sector no socialista es la existencia de un sector socialista, en particular aquella parte del sector socialista que está nacionalizado" (1976: 21). En una primera fase, el control de la economía según Lange (1976) tenderá a ser altamente centralizado en aspectos como la planeación, dirección y administración.

Ahora bien, en la "planificación socialista", según el Manual de Economía Política de la URSS, seguirá rigiendo la "ley del valor". Lo anterior significa que en el valor de las mercancías seguirá rigiendo lo expuesto anteriormente por Karl Marx, pero los precios serán fijados por el gobierno de acuerdo a la "ley del valor". De esta manera, el valor de las mercancías sería igual al valor de los medios de producción que se transfiere al valor de las mercancías producidas (depreciación, materias primas, entre otros.) y por el nuevo valor creado (valor del producto necesario utilizado para remunerar el trabajo y el valor del producto nacional que se destina a satisfacer las necesidades, es decir, pasa a formar parte de los ingresos de las empresas estatales y la renta del Estado). De esta manera, el precio planificado debe correlacionar oferta y demanda y debe ser superior al valor de las mercancías para que la producción sea rentable (AC-URSS, 1975), para asignar administrativamente remuneraciones a factores y es el mecanismos de asignación de recursos en el modelo de "planificación socialista".

No obstante, en este sistema económico el dinero cumple la función de medida del valor, medio de pago en el comercio, de "acumulación socialista", instrumento de planificación, control de la producción o distribución del "producto social". Por lo tanto, la transformación del valor en precio en el socialismo planificado centralmente transita la asignación administrativa de precios por parte del Estado.

La planificación centralizada implementada por los países socialistas y que se concretó en los denominados planes quinquenales, según Gregory y Stuart (1989) siguió enfrentando serios problemas: los problemas concretos tales como: de asignación de recursos; establecimiento de incentivos que surgen de los resultados de la aplicación del plan; problemas de coordinación y cronogramas de aplicación de recursos y esfuerzos para encauzar diferentes y numerosos proyectos sobre todo en el ámbito agrícola e industrial; la sustitución de la competencia por la "emulación socialista" no ha probado ser un acicate adecuado para motivar el incremento de la productividad en las empresas aunque cuenten con tecnología avanzada. Estos problemas fueron abordados por los teóricos del "socialismo de mercado".

Los antecedentes teóricos del socialismo de mercado se pueden encontrar en, según Gregory y Stuart (1989), en 
Enrico Barone, Janos Kornai, Oskar Lange y Jaroslav Venek entre otros.

En su obra II Ministerio della Produzione nello Stato Collettivista, Enrico Barone considera una sociedad en que algunos capitales serán de propiedad privada (tales como los bienes personales) y otros capitales serán de carácter colectivo (capital fundacional y el capital mobiliario). Dada la existencia de una propiedad mixta, el principal problema del ministro de la producción es la combinación de tales propiedades con la finalidad de maximizar el bienestar a sus administrados; es decir, con sus palabras: "Il ministro della produzione ha da risolvere il problema di combinare quiesti servizi individuali e collettivi in guisa da procurare il maggior benessere ai suoi amministrati" (Barone, 289). El ministro de la producción parte de una economía constituida solo de bienes y en donde, por razones de contabilidad social (Contabilitá sociale), establece lo que él denominó como relaciones de equivalencia (Rapporti di equivalenza). Estas relaciones de equivalencia (que denominó ë) entre objetos producidos e intercambiados serán establecidas por el ministro (mediante el establecimiento de un comité de planificación central o Ministero della Produzione y de oficinas contables para el registro y control de las operaciones de producción y distribución según el marco legal establecido) principalmente en los productores de carácter colectivo propiedad del Estado. Del total de la producción $\left(Q_{1} \lambda_{1}+Q_{2} \lambda_{2}+\ldots+Q_{n} \lambda_{n}=X\right)$ ajustada a las relaciones de equivalencia, podrán estar disponibles directamente (forma preferida por el ministro de la producción) o indirectamente (disminuyendo las equivalencias del producto) para el uso de la población. Esa distribución, según Baro- ne se establece entre el ministro y los administrados, perfeccionado bajo la figura jurídica de una legislación positiva. Esa distribución consensuada debe garantizar que parte del consumo se destine al ahorro y la inversión para reponer e incrementar el monto del capital colectivo. Sin embrago, el ministro tendría la prerrogativa de imponer cuotas de ahorro mayores (de menor consumo) a determinados individuos. En el caso de que determinados grupos sociales no contribuyan con mayores cuotas de ahorro, el ministro podría eventualmente extraer ahorros de parte de la producción total, establecer incentivos económicos para quienes difieran para el futuro su consumo o establecer una política de préstamos al Estado a determinada tasa de interés.

Para Kornai (1979), desde el punto de vista del análisis microeconómico, el incremento de la producción enfrenta tres restricciones efectivas (realmente limitan la elección): 1) la disponibilidad de recursos; 2) restricciones de la demanda; y 3) restricciones presupuestales. Por tanto, desde el punto de vista de la efectividad de las restricciones, el sistema capitalista tiene restricciones de demanda y de presupuesto y el sistema socialista de producción. En el caso de los sistemas sometidos a restricciones de recursos, según Kornai (1979), la tecnología está dada, con coeficientes de insumo-producto estables y cuando existen cuellos de botella los recursos pueden permanecer ociosos. Esos cuellos de botella surgen por la escasez de los recursos necesarios. Por lo tanto, según Kornai (1979), la escasez y la inactividad no son fenómenos mutuamente excluyentes. 
Con respecto a las restricciones de carácter presupuestario, según Kornai (1979), la planificación económica socialista, que se sustenta en el establecimiento de metas de expansión de la oferta, tiende a generar un proceso presupuestario blando en donde queda en un segundo plano la atención a los precios y la cobertura de costos. La empresa capitalista, por medio del mecanismo de precios, busca maximizar la utilidad. De esta manera, la existencia de restricciones presupuestarios desincentiva la sobredemanda de insumos. Es decir, la empresa capitalista solamente gasta lo que tiene permitido, demanda insumos en función de sus precios de mercado y la situación financiera de la empresa, la obtención de crédito se presenta bajo condiciones de mercado bancario generalmente conservadoras.

Las propuestas de Kornai (1979) también abarcan aspectos de carácter macroeconómico. En el ámbito de la economía nacional, de manera simplificada, propuso un modelo con un sector de producción de bienes de producción, de producción de bienes de consumo, depósitos para bienes de consumo y otro para bienes de producción. En lo referente al depósito de bienes de consumo existe una relación entre los flujos de entrada y salida asociados al ingreso nominal de las familias y el nivel agregado de precios. Cuando el ingreso nominal se incrementa y cae el nivel agregado de precios, se produce un incremento de los flujos de salida que debe ser compensado por los flujos que entran al depósito de bienes de consumo. De existir restricciones en la producción y consecuentemente en los flujos de entrada en el depósito de bienes de consumo, se presentan problemas de escasez y eventualmente de acaparamiento que amplifica el fenómeno de la escasez. Lo anterior llevará a las autoridades, en un ambiente de restricción presupuestaria blanda a incrementar las metas de producción y presionar en búsquedas de recursos financieros (apetito por la inversión [Kornai, 1979]) y materiales.

Ahora bien, para Kornai (1979) en una economía monetizada, la inflación sería el elemento de corrección a la demanda creciente de bienes y servicios (efecto saldos reales [Ekelund y Hébert, 1992]). Por lo tanto para controlar el exceso de demanda agregada (que eventualmente afiance la escasez), en un ambiente con restricciones presupuestarias duras para empresas y familias y con política de control de precios (inflación reprimida), se debe abrir el camino para el incremento en los precios. No obstante, según Kornai (1979), la existencia en la economía de empresas o sectores económicos con restricciones presupuestarias blandas, no tienen incentivos negativos para ajustarse a las subidas de precios por medio de la reducción de su demanda. La empresa o sector económico no restricción presupuestaria blanda podrá transferir al resto de los sectores los costos adicionales incurridos en sus procesos de producción. Por lo tanto, la supervivencia de las empresas o sectores económicos con restricciones presupuestarias blandas dependerá de subsidios por parte del Estado o trasladar a la sociedad vía impuestos o endeudamiento público (interno o externo) parte del costo de funcionamiento.

La propuesta de Oskar Lange parte de exponer un modelo que parte del es- 
quema de equilibrio similar al de Pareto, Walras o Barone (1859-1924) (Gregory y Stuart, 1989) con la finalidad de lograr su solución atravesando diferentes etapas dentro de un esquema de "solución competitiva" que se corresponde con el modelo de competencia perfecta (Bergson, 1967). Este autor propuso tres niveles de decisión: las del nivel inferior (las empresas, las familias), el nivel intermedio conformado por las autoridades industriales, y el Comité de Planificación Central.

En el nivel central, se establece los precios de los bienes a producir y la asignación de los dividendos sociales (diferencia entre las ganancias de las empresas menos los intereses y rentas pagados por ellos) (Bergson, 1967 y Gregory y Stuart, 1989). Según Bergson (1967), los precios fijados por el Comité Central de Planificación son informados a las empresas productivas y se les instruye para producir de acuerdo a las siguientes reglas: 1) el nivel de producción será aquel en que el precio se iguale al costo marginal; y 2) que a ese nivel de producción se minimice el costo. El nivel de las familias se les otorga la libertad en las decisiones de oferta de mano de obra. Ahora bien, esta primera asignación de precios puede ser o no cumplida por las empresas y eso pudiese generar desbalances entre oferta y demanda. Cuando se presenta sobreoferta de bienes y servicios, en un segundo periodo, el Comité Central de Planificación puede optar por reducir el precio y viceversa. Por otra parte, la distribución de las rentas y ganancias sería realizada bajo la forma de servicios e inversión. Por otra parte, el Comité de planificación central decide los volúmenes de inversión y las tasas de interés reque- ridos para financiar el crecimiento de la infraestructura económica.

Según Bergson (1967), uno de los problemas que atraviesa la implementación de la solución competitiva propuesta por Oskar Lange radica en que no estableció algún criterio para juzgar el éxito o no de las decisiones de la gerencia.

La variante cooperativa o de "economía participativa" impulsada por Jaroslav Vanek (1970) y Benjamín Ward (1958) se caracteriza por: 1) las empresas pueden tomar decisiones de producción y de administración de manera participativa; 2) la economía funcionaría en un modelo de mercado, la planificación sería de carácter indicativo; 3 ) existe la libertad de elección del empleo; 4) la distribución del ingreso tenderá a ser equitativa y democrática, es decir, igual ingreso para igual intensidad y calidad de trabajo; 5) hace uso del análisis marginal.

De esta manera, los recursos son propiedad del Estado, la administración se basa en cooperativas y los productos (medios de producción y productos finales) son intercambiados en el mercado sin la intervención de los planificadores económicos.

En el corto plazo, el modelo cooperativo asume que los administradores buscan maximizar la ganancia neta por trabajador (Y/L) y que la producción Xt depende solamente del factor trabajo, es decir, $X t=f(L)$. $X t$ se vende libremente en el mercado a un precio $P$ t. Por lo tanto $Y / L$ $=P t X t / L$. la maximización de $Y / L$ se dará cuando $L$ alcance un punto en que el valor del producto marginal del último trabajador empleado sea equivalente a la ganancia neta por trabajador, esto es, 
Pt.PmgL $=Y / L$, donde $P m g L=$ productividad marginal del trabajo. Según este esquema, la cooperativa puede contratar más empleados hasta cierto límite que le permita ser factible económicamente. En el largo plazo, donde la cooperativa debe velar por un nivel óptimo de capital, se debe pagar un interés por unidad de capital empleado $r$ (que es equivalente a Pt.PmgK o productividad marginal del capital). De esta manera, la cooperativa busca ahora maximizar $Y / L=P t . X t-r$ $(K) / L$, donde $K$ es el monto de capital empleado. Por tanto, la maximización de $Y / L$ se dará cuando $L$ alcance un punto en que el valor del $P m g L$ del último trabajador empleado sea equivalente a la ganancia neta por trabajador ajustado al monto y el interés por unidad de capital empleado. Si Pt.PmgK > r, mayores unidades de capital serán adquiridas.

Entre las ventajas que Vanek (1977) vio en su modelo de cooperativismo se encuentran: 1) es ideal para las economías del tercer mundo; 2) si las cooperativas cuentan con idéntica estructura tecnológica y cuentan con libertad de entrada y salida en el mercado, tienen un desempeño similar a la empresa capitalista; 3) De acuerdo a lo anterior, la cooperativa, según Vanek está en mejor posición que la empresa capitalista debido a que la contratación de nuevo personal surge de acuerdo entre los socios y no de la PmgL; 4) la cooperativa competitiva imperfecta es superior a su equivalente sociedad anónima, debido a que no tiene incentivo de crecer demasiado y de adueñarse de porciones cada vez más grandes del mercado.

\section{Aproximación a una caracterización del "Socialismo del Siglo XXI"}

En el V Foro Social Mundial celebrado en 2005, el Presidente Hugo Chávez Frías hizo mención del "Socialismo del siglo XXI" en el marco de la "Revolución Bolivariana" y que para llegar a esta etapa se debe transitar lo que él denominó como "Democracia Revolucionaria" (Zambrano, 2008). Según María Luisa Zambrano en otra alocución realizada en 2006 el presidente Chávez expresó: "Hemos asumido el compromiso de dirigir la Revolución Bolivariana hacia el socialismo y contribuir a la senda del socialismo, un Socialismo del Siglo XXI que se basa en la solidaridad, en la fraternidad, en el amor, en la libertad y en la igualdad" (2008: 28).

Alguno de los antecedentes de las propuestas del "Socialismo del siglo XXI" aparecen esbozado en documentos, por ejemplo, en "Agenda Alternativa Bolivariana" (Movimiento V República, 1996) en donde las empresas básicas tendrían un régimen de propiedad estatal, los bienes de consumo esencial, los servicios esenciales y gobierno un régimen de propiedad mixto, la gran industria un régimen de propiedad privada y la banca un régimen mixto con regulación pública. Posteriormente, en la propuesta de reforma constitucional rechazada en 2007, se esbozó de manera más abierta la necesidad de establecer un modelo de economía socialista, especialmente en sus artículos 122, 156, 299, 300, 321, la disposición transitoria donde se establece la legislación de las siguientes leyes: Ley para la 
Promoción de la Economía Socialista, Ley de Reforma del Banco Central de Venezuela, Ley Especial del Fondo Nacional del Poder Popular entre otras leyes.

Otros quienes han contribuido a extender el discurso del "Socialismo del Siglo XXI" son las propuestas de "economía equivalente" de Heinz Dietrich ${ }^{4}$. En una economía equivalente: "solo se producirán solamente bienes para cubrir necesidades, y serán consumidos por el productor, o se canjearán al mismo valor (base de la distribución en la economía equivalente" (Dietrich, 2007: 117). De esta forma, para Dietrich:

"El salario equivaldrá al tiempo de trabajo invertido, independientemente de la edad, del sexo, del estado civil, del color de la piel, de la nacionalidad, del tipo de trabajo, del esfuerzo físico, de la preparación escolar, del desgaste, de la habilidad, de la experiencia profesional, de la entrega personal al trabajo, independientemente también, de la pesadez del trabajo y de los peligros que impliquen para su salud. En pocas palabras: el salario equivale directa y absolutamente al tiempo laborado. Los precios equivalen a los valores, y no contienen otra cosa que no sea la absoluta equivalencia del trabajo incorporado en los bienes" (Dietrich, 2007: 109).

En el esquema de Dietrich (2007), el comercio se limitará a la distribución de bienes y se remunerará en función del tiempo de trabajo invertido. En este esquema, siguiendo a Arno Peters, Dietrich
(2007) propone la desaparición de la ganancia y el mercado.

Por otra parte, Dietrich (2007), expuso para que el nuevo socialismo garantice el derecho a vivir y evolucionar dignamente se requiere de la "democracia participativa", utilizando los mecanismos plebiscitarios por medio de la Internet. Además, el Estado, al igual que en el comunismo no ejercerá su función de representante de clase y solamente existirá como órgano administrativo. Todo lo anterior permitirá que se desarrollen en el ser humano: "el pensamiento crítico-racional, su capacidad estética (artística) y su potencial ético (moral) (Dietrich, 2007: 139).

Para nosotros el "Socialismo del siglo XXI", al menos desde el punto estrictamente económico, se encuadra en un esfuerzo de instaurar en Venezuela el denominado "socialismo de mercado".

En efecto, desde nuestro punto de vista, la economía venezolana a partir de 1999 está atravesando un proceso lento de implantación del esquema de Enrico Barone, de Oskar Lange y de Jaroslav Vanek en los siguientes términos: 1) La compra o renacionalización de empresas de Commodities y de Utilities y transformarlos en la base del sector socialista de la economía. 2) el establecimiento de metas de producción y precios (de adquisición de insumos y de venta al público) siguiendo normativas del Poder Público Nacional e implementados por las empresas del sector socialista; 3 ) los desbalances entre oferta y demanda se han cu- 
bierto con subsidios cambiarios, a la producción y al consumidor con cargo al presupuesto fiscal y, a su vez, con cargo a la renta petrolera; 4) la creación de organizaciones dedicadas a la oferta de bienes y servicios ha sido, en muchas ocasiones, por medio de créditos blandos otorgados por instituciones financieras al margen del control de las autoridades reguladoras, con la finalidad de generar rápidamente organizaciones de propiedad social en diferentes ramas de la producción; 5) la existencia de ministerios que atiendan a la economía social recoge lo que planteó en su momento Enrico Barone con su Ministero della Produzione; 6) la construcción de un discurso que condena la ganancia capitalista; 7 ) implementación de un vasto plan de asistencia pública a la población de bajos recursos materializado en las llamadas "misiones"; 8) el intercambio mercantil sobre la base del valor; 9) hacer recaer el peso del proceso económico en el nivel comunal concentrados en Consejos Comunales, Comunas, Núcleos de Desarrollo Endógeno o ciudades Socialistas; o 10) presión regulatoria al sector privado de la economía en materia cambiaria, fiscal, regulación de precios, regulación de salarios o inamovilidad laboral.

\section{Propuestas de Mises expuestas en Bureaucracy}

El principal aporte de Mises radica específicamente en movilizar las representaciones sociales de burocracia, resemantizarlo.

Mises reconoce que las palabras burócrata, burocrático y burocracia son términos claramente construidos, con connotaciones negativas tanto por las teorías de inspiración capitalista como aquellas de inspiración socialista (Mises, 1983[1944]) $y$, según el autor antes mencionado, ya se hablaba de ella desde los imperios egipcios, chino, la Francia borbónica o la Prusia de Federico II el Grande ${ }^{5}$; sin embargo, llama la atención acerca de lo que realmente significa burocracia.

Aún cuando cierto grado de burocracia es indispensable en la sociedad, ésta se convierte en algo pernicioso, cuando forma parte de un Estado totalitario. La razón para esta afirmación la expresa Mises de esta manera: "El totalitarismo es mucho más que la mera burocracia. Es la subordinación de la vida, ocupación y tiempo libre del individuo a la orden de quienes detentan el poder. Es la reducción del hombre a ser un engranaje de la maquina de coerción" (1983[1944]: 18). Es decir, la burocracia se convierte en un instrumento de ejecución de los objetivos de quienes la controlan.

Ahora bien para terminar de comprender qué es realmente la burocracia, es necesario entender cómo funciona la economía capitalista. Es decir, qué es lo que la burocracia quiere someter bajo su dominio. En forma esquemática la forma de operar de un sistema de mercado es la siguiente según Ludwig von Mises.

1. Es un sistema de cooperación social y división del trabajo, que permite aplicar métodos de cálculo económico para el desarrollo de nuevos proyectos y trabajar a plena capacidad de tal manera que los ingresos cubran los costos.

2. Se basa en la propiedad privada de los medios de producción.

3. Los factores materiales de producción son propiedad de individuos, los 
capitalistas y los propietarios de la tierra.

4. El empresario libre tiene como objetivo la ganancia.

5. Lo que producen los empresarios es para el mercado.

6. Los empresarios pueden tomar decisiones en base a sus objetivos, pero no son libres de determinar el curso del mercado (Mises, 1983[1944]: 23). El personaje central en la vida del mercado es el consumidor.

7. Uno de los problemas a ser resueltos es qué producir y con qué procedimientos producir de la manera más ventajosa con la existencia de innumerables y diversos tipos de factores de producción. Una forma de resolver éste problema es utilizando el sistema de precios. En términos de Mises: "La guía de la planeación económica es el precio de mercado" (1983[1944]: 26).

8. Sin embargo, no se debe considerar a los precios del mercado como un valor absoluto. Los precios de mercado apunto Mises (1983[1944]) dependen de las preferencias subjetivas de los agentes (actores) económicos que en él participan. Es decir: "Los juicios de valor son el resultado de la arbitrariedad humana" (Mises, 1983[1944]: 32).

El término burocracia fue introducido por primera vez porJean-Claude Marie Vicent de Gournay (1712-1759), el mismo que introdujo la famosa consigna liberal "Laissez-Faire, Laissez Passer". Uno de quienes reflexionaron inicialmente con gran profundidad el tema en el siglo XIX y XX fue Max Weber. Para Weber (1977[1922]) existen tres tipos puros (Idealtypen) de dominación legítima: 1) la racional, basada en la creencia y en la legalidad de las normas aprobadas y en los consecuentes derechos de mando que generan autoridad que puede ser ejercida por quien sea su titular (autoridad legal); 2) la tradicional que descansa en la creencia cotidiana y en las tradiciones y que general la autoridad tradicional; y 3) la carismática: "Que descansa en la entrega extracotidiana a la santidad, heroísmo o ejemplaridad de una persona y a las ordenaciones por ella creada o reveladas (llamada) (autoridad carismática) (Weber, 1977[1922]: 172). En este punto interesa destacar la dominación legal en presencia de la administración burocrática. La dominación legal se puede establecer por: 1) un derecho vigente estatuido de modo racional con arreglo a fines o a valores; 2) tal derecho se aplica a casos concretos de acuerdo; 3) quien aplica la norma obedece el orden impersonal que establece la norma vigente; 4 ) quien obedece lo hace a la norma no a la persona que aplica la norma, dentro de una competencia limitada, racional y objetiva. En el punto (4), las categorías fundamentales de dominación legal son, según Weber (1977[1922]): a) el ejercicio continuado; b) dentro de una competencia; c) en presencia de una jerarquía administrativa u ordenación de autoridades; d) con reglas que pueden ser técnicas o normas; e) la jerarquía funciona con atención al principio de separación entre el cuadro administrativo y la remuneración y rendición de cuentas; f) no se presenta la apropiación de los cargos; g) rige el principio administrativo de atenerse al expediente $\mathrm{h}$ ) según Weber: "El tipo más puro de dominación legal es el que se ejerce mediante un cuadro administrativo burocrático" (1977[1922]: 175); h) la calificación profesional que fundamenta el nombramiento requiere de una serie de evaluaciones de certificación; h) ser retribuidos con dinero, bajo la forma de sueldos y pensiones; i) quien ejerce el cargo como única o principal profesión; j) su ascenso o promoción implica la idea de carrera administrativa, con separación de los medios administrativos y sin apropiación del cargo; y k) están sometidos a la disciplina e inspección administrativa. 
9. Por lo tanto, las ganancias en el sistema capitalista, según Ludwig von Mises, provienen del hecho de que la sociedad no es estacionaria.

10. Para que los negocios funcionen con cierta continuidad, el empresario, capitalista o granjero debe contar con especialistas en planificación financiera, contadores y depender de un estructura organizacional a cargo de personal especializado en tareas de dirección y liderazgo organizacional. En este sistema la responsabilidad en la búsqueda de ganancias se encuentra dividida horizontalmente y verticalmente. Esto nos lleva a las ideas de delegación, descentralización y responsabilidad en la toma de decisiones. "El propio interés empuja al gerente a cuidar el resultado en su propia sección. Si él incurre en pérdidas, él será reemplazado por otro hombre del que el gerente general espera más éxito" (Mises, 1983[1944]: 37).

Ahora bien, el sistema de libre mercado admite la existencia de la burocracia, afirma Mises; sin embargo, la burocracia se encuentra sometida a la democracia que es lo mismo que decir la supremacía de la ley y del control presupuestario (Mises, 1983[1944]: 47). Luego la ley debe ser diseñada con la finalidad de establecer límites al poder de las autoridades y proteger los intereses de los ciudadanos como acreedores de derecho/deberes y de su papel como consumidores/productores. Estas importantes tareas llevaron a Ludwig von Mises a distinguir entre las tareas que realiza una gerente burocrático de uno en el mundo de los negocios (Ver Cuadro 1).
Si la gerencia pública tiene tantos problemas para ser vigilada y además se le considera ineficiente lenta, ¿por qué no adopta los métodos probados de la administración privada? Precisamente respondería Ludwig von Mises debido a que la administración pública no tiene como fin las ganancias y sus fuentes de financiamiento son facilitadas por ser el portador de la soberanía del Estado. Pero además, la calidad de un empresario no depende de su personalidad como empresario, sino que es inherente a la posición que ocupa en la estructura de mercado de la sociedad. Mientras que la calidad del burócrata no depende de su papel dentro de la estructura de mercado, sino de su capacidad de cumplir y hacer cumplir leyes, normas, regulaciones o reglamentos. Por eso dice Mises: "Es una ilusión muy popular el hecho de que la eficiencia del gobierno puede ser mejorada por ingenieros de la gerencia y sus métodos de gerencia científica" (1983[1944]: 54).

Una vez que Ludwig von Mises analizó las burocracias tanto en el sector público como en el privado, se dedicó a estudiar las características y comportamiento de la burocracia en un sistema socialista y en un sistema de libre empresa.

Mises caracteriza a la burocracia socialista: "Como el control gubernamental de todas las actividades económicas y en donde existe carencia del instrumento intelectual indispensable para la planificación y diseño económico: el cálculo económico" (Mises, 1983[1944]: 62). Para Mises, la negativa a considerar el sistema de precios de mercado es lo que ha llevado al socialismo al caos. Mises considera que esa negativa ha convertido el tema de los precios como uno de los 


\section{Cuadro 1 \\ Diferencias entre la gerencia burocrática y empresarial}

\begin{tabular}{|c|c|c|}
\hline No. & Gerencia empresarial & Gerencia burocrática \\
\hline 1 & $\begin{array}{l}\text { Tiene como motivo la obtención } \\
\text { de ganancias }\end{array}$ & $\begin{array}{l}\text { No tiene como motivo principal la generación de } \\
\text { efectivo y la ganancia. }\end{array}$ \\
\hline 2 & $\begin{array}{l}\text { Es muy importante la relación entre } \\
\text { los ingresos y los costos marginales. }\end{array}$ & No hay conexión entre ingresos y gastos. \\
\hline 3 & Debe ajustar sus gastos a los ingresos & $\begin{array}{l}\text { La administración pública solamente gasta di- } \\
\text { nero. }\end{array}$ \\
\hline 4 & $\begin{array}{l}\text { Sus ingresos provienen del mercado o } \\
\text { el endeudamiento por vía de bonos o } \\
\text { préstamos. }\end{array}$ & $\begin{array}{l}\text { Sus ingresos provienen de los impuestos, el en- } \\
\text { deudamiento o la impresión de dinero. }\end{array}$ \\
\hline 5 & $\begin{array}{l}\text { Sus ingresos son una variable aleato- } \\
\text { ria, el consumidor, en que además } \\
\text { desconoce su estructura de ingresos }\end{array}$ & $\begin{array}{l}\text { La fuente de sus ingresos por vía fiscal lo facili- } \\
\text { ta la ley. Es decir, el recaudador no tiene méri- } \\
\text { tos al recaudar algo que le establece la ley }\end{array}$ \\
\hline 6 & Tiene precio o valor de mercado. & No tiene precio de mercado por sus logros. \\
\hline 7 & $\begin{array}{l}\text { Su vigilancia y control se facilita por } \\
\text { el cálculo económico }\end{array}$ & $\begin{array}{l}\text { La gerencia burocrática es la gerencia que no } \\
\text { puede ser vigilada por medio del cálculo econó- } \\
\text { mico. }\end{array}$ \\
\hline 8 & $\begin{array}{l}\text { El mercado de valores o de crédito va- } \\
\text { lora el desempeño de la empresa en } \\
\text { términos de riesgo de negocios o pre- } \\
\text { cio de una acción. }\end{array}$ & $\begin{array}{l}\text { El resultado de la acción gubernamental no tie- } \\
\text { ne precio en el mercado. }\end{array}$ \\
\hline 9 & $\begin{array}{l}\text { Depende de los resultados en térmi- } \\
\text { nos económicos, reducción de costos, } \\
\text { mayores ventas. }\end{array}$ & $\begin{array}{l}\text { La promoción depende de los años de servicios } \\
\text { y las promociones que están implícitas. La ma- } \\
\text { yor parte del tiempo ocupa labores subordina- } \\
\text { das, siguiendo reglas preestablecidas. }\end{array}$ \\
\hline 10 & $\begin{array}{l}\text { Hay más opción para la creatividad, la } \\
\text { innovación, porque el premio son ma- } \\
\text { yores ganancias }\end{array}$ & $\begin{array}{l}\text { Por lo anterior, existe poco incentivo para la } \\
\text { creatividad, la inventiva y atreverse a cuestio- } \\
\text { nar procedimientos. }\end{array}$ \\
\hline 11 & $\begin{array}{l}\text { Servir a la comunidad y obtener ga- } \\
\text { nancias. }\end{array}$ & $\begin{array}{l}\text { La principal tarea de la administración consiste } \\
\text { rendir servicios útiles a la comunidad. }\end{array}$ \\
\hline
\end{tabular}

Fuente: Mises (1983[1944]: 50, 51).

problemas más grandes que debe enfrentar el marxismo en todas sus variantes (Mises, 1983[1944]: 63). Este problema se presenta en la administración de empresas propiedad del Estado en el mundo occidental. Cuando una empresa pública tiene pérdidas, la capitalización de la empresa se hace vía presupuesta- ria, bien sea con aumento de los impuestos, las tarifas o el endeudamiento. En otras palabras, lo que ahorra un consumidor por vía de los subsidios directos o indirectos, se gasta en el incremento de los impuestos o la tasa de interés (Mises, 1983[1944]: 65). 
Si bien es cierto que la empresa pública busca ofrecer servicios útiles a la comunidad, el problema consistirá en ¿Quién decide lo que es útil para la comunidad? O si algún servicio es útil o no y mucho más importante: ¿Cómo saber si un determinado servicio público tiene un costo de realización alto, si los factores de producción absorbidos para realizar el servicio son sacados de otros servicios que desarrolla el organismo público y que pueden ser más útiles en su empleo? (Mises, 1983[1944]: 65). En el sector privado, esta pregunta se responde por medio de las actitudes positivas o negativas del público. Sin embargo, en el sector público, el comportamiento del público no provee un criterio certero de la desutilidad ó utilidad del servicio ofrecido y tampoco el burócrata es responsable por el resultado.

Una vez hecha la caracterización de la burocracia tanto en los sistemas capitalistas y socialistas, pasó a analizar los fundamentos de la filosofía del burocratismo y las consecuencias psicológicas.

Los conflictos políticos, según Ludwig von Mises, surgen de la batalla entre dos principios, el bien y el mal (Mises, 1983[1944]: 81). Para Mises, la filosofía del burocratismo o como él la denominaba la "Philosophy of Statolatry", visto desde la superficie, se reduce a lo siguiente: "El bien está introducido en el seno del Estado, la materialización de la eterna idea de moralidad y que lo malo es patrimonio del individualismo. En este antagonismo, el Estado personifica el bien y el individuo el mal; el Estado es representante de la justicia, la civilización y el juicio superior" (Mises, 1983[1944]: 81). Ahora bien, la esencia de la filosofía del burocratismo es: "Las leyes escritas que, ante los ojos de los ad- ministradores, se constituyen en barreras levantadas por personas de mal proceder en contra los justos reclamos de la sociedad" (Mises, 1983[1944]: 82). Sin embargo, Mises rechaza la idea que busca eliminar leyes imperfectas, ya que eso sería la vía más expedita al totalitarismo. Por esta razón afirmó que: "La peor ley es mejor que una tiranía burocrática" (Mises, 1983[1944]: 83). Por eso la elaboración de las leyes no puede dejarse a merced de algunos hombres. Las leyes deben ser diseñadas para convertirse en leyes basada en reglas, ya que: "La alternativa a las leyes basadas en reglas son las reglas basadas en el despotismo" (Mises, 1983[1944]: 83).

\section{Propuestas de Mises expuestas en The Human Action}

En esta obra Mises precisa más aún las ideas que sustentan la forma socialista de hacer las cosas en materia de gerencia pública y en tal sentido dice: "A socialist advocates socialism because he is fully convinced that the supreme dictator of the socialist Commonwealth we will reasonable From his point of view, that he will aim at those ends of which he fully approves, and that he will try to attain these ends by choosing means which he would also choose" (1996[1949]:670671). Es decir, el gerente o dirigente socialista esta en capacidad de decidir qué es lo que la sociedad necesita y sabe como obtener los medios para lograr los fines sociales. Por esta razón Mises más adelante plantea la necesidad de que en el régimen administrativo socialista se establezca o imponga lo que él denominó 
como Gleichshaltung, es decir, la uniformidad de criterios o de pareceres. Para Mises, el socialismo es otra forma de religión con sus deidades, en la cual, por ejemplo, "Para los planificadores es el estado o el gobierno, para los nacionalistas es el pueblo, para los marxistas la sociedad o para el positivismo de Comte es la humanidad" (1996[1949]:671). Para Mises, estas deidades, no son: "sino meros nombres que contiene solamente los deseos del reformador" (1996[1949]:671).

Según Mises (1996[1949]), existen dos formas para la realización del socialismo: 1) los que él denominó como el modelo ruso; 2) el modelo alemán. El modelo ruso se sustenta en las nacionalizaciones (verstaatlicht), en que los departamentos del gobierno es operado por "sirvientes civiles" (1996[1949]: 697) y en que cada unidad del aparato establece con su centro una relación de subordinación. El modelo alemán, preserva la propiedad privada sobre los medios de producción y mantiene la apariencia de una economía de mercado; sin embargo, existen pocos empresarios que conviven con funcionarios gubernamentales que Mises denominó como los Betriebsführer. Estos funcionarios: "Compran, venden, contratan, despiden trabajadores y remunera sus servicios; contratan préstamos, pagan los intereses y la amortización de los créditos. Sin embargo, todas sus actividades se basan en la obediencia incondicional de la oficina suprema de gerencia de pro- ducción del gobierno, que en la terminología nazi se le denomina Reichswirtschaftsministerium" (Mises 1996[1949] 697-698). Este ministerio de la economía le establece a sus funcionarios: 1) qué y como producir bienes y servicios; 2 ) a qué precios y la forma de distribución de esos bienes y servicios; 3 ) establece la remuneración a los trabajadores; 4) establece en qué términos los capitalistas deben confiar sus recursos al gobierno; 5 ) el mercado cambiario es controlado; y 6 ) el gobierno dirige todas las actividades de producción. En este sistema los funcionarios, supervisores y gerentes están sujetos al gobierno, no a la demanda de los consumidores y la estructura de precios del mercado ${ }^{6}$.

Ahora bien, como el socialismo, según Mises, se sustenta en la creencia de que el socialismo es inevitable, surgido de un progreso continuo de la sociedad y que esta es un ser omnisciente, libre de las debilidades y fragilidades humanas, no considera de quienes ejercen funciones de liderazgo político o de administrador público, son seres humanos con defectos y falibles y no una "entidad mítica colectiva" (Mises, (1996[1949]:674). El hecho de que un gerente en el socialismo dispone de todos los medios tecnológicos, humanos y materiales necesarios lo obliga a tomar decisiones dentro de una infinita variedad de proyectos. Es posible que deba dedicar recursos a proyectos que considera no son prioritarios para sa-

6 Según Mises: "This is socialism under outward guise of the terminology of capitalism. Some labels of the capitalistic market economy are retained, but they signify something entirely different from what they mean in the market economy. (1996 [1949]: 698). 
tisfacer necesidades sociales y viceversa. Es decir, la actuación del gerente en este sistema no necesariamente tiene que ver con la valoración con relación a los fines últimos de la sociedad. Según Mises, se refiere solamente a los medios empleados para alcanzar los fines últimos.

Si el problema es acerca de los medios para alcanzar fines dentro de un marco en donde el mercado no es referente en materia de precios o la ganancia no es un criterio de toma de decisiones, aunque un gerente en el socialismo puede contar con infinidad de recursos materiales y humanos para desarrollar iniciativas puede establecer cualquier combinación de factores. El problema no es establecer cualquier combinación de factores, sino aquella combinación que permita cumplir con sus objetivos con los recursos disponibles. Esto es lo que Mises denomino como "calculo económico". Se debe establecer una iniciativa que en términos de costos, tiempo y calidad del resultado peritan cumplir con los fines planteados.

Mises acepta la crítica socialista de que el más elaborado cálculo económico puede presentar serias fallas si las expectativas referidas al futuro no coinciden con la realidad. Para Mises, los gerentes, en el sistema capitalista, solo hacen sus cálculos económicos a la luz del conocimiento presente y la anticipación también en el presente de condiciones futuras de la situación económica-social; es decir, el problema no se trata de si el gerente tiene habilidades o no de predecir el futuro. Lo que Mises trata de expresar es que el gerente en el sistema socialista no puede calcular desde el punto de vista de su propio presente y de su juicio acerca del futuro. Su problema con el futuro, quizá, sea similar al del gerente en el sistema capitalista; pero su problema con el presente es la inexistencia de un sistema de precios que le permita, ya no tomar la "decisión óptima", sino una buena decisión en términos de costos y con relación a los recursos disponibles. Porque si el socialismo busca satisfacer necesidades sociales prioritariamente, un sistema que tienda al desperdicio por falta de cálculo económico será aquel que contaría con menores recursos para emprender nuevas iniciativas. Pensar en planificación (indicativa o centralizada) sin cálculo económico es suponer que los bienes son "libres". Si los bienes son libres entonces, no habría cálculo económico y mucho menos Economía. Por esta razón, Mises plantea la "paradoja de la planificación", ya que en ausencia de cálculo económico no puede haber plan. Más aún, la gerencia solamente serviría para hacer cumplir lo decidido en instancias extraeconómicas y reproducir el orden de cosas existentes. Mises (1996[1949]) ha expuesto que los defensores del socialismo han intentado: 1) introducir el cálculo económico con base de bienes por sobre el dinero; 2) utilizar la hora de trabajo como unidad de medida; 3) el establecimiento de lo que denominó como "Quasi-market"; 4) aplicar el método del ensayo y el error. A pesar de todas estos arreglos, para Mises: "el problema del calculo económico socialista es este: que en ausencia de precios de mercado para los factores de producción, un computo de ganancias o pérdidas no es factible" (1996[1949]: 684). Según Mises (1996[1949]): 1) es como desfragmentar lo fragmentado, es decir, sumar o restar cosas de diferente naturaleza y origen; 2 ) 
no se considera las diferentes cualidades del trabajo, los diferentes orígenes de los insumos y que las productividades laborales para, incluso una misma, tarea son diferentes; 3) para Mises es paradójico que un sistema que quiere eliminar el mercado y la propiedad privada y al mismo tiempo establecer algún tipo de mercado, ya que la existencia misma del mercado refuta al socialismo ${ }^{7}$; y 4 ) esto es solo aplicable en todos los casos en el cual la solución correcta es reconocible y no depende de la aplicación del método en si.

Mises tanto en Bureaucracy y en Human Action reconoció la existencia de un sistema de administración pública que no es precisamente socialismo. Dentro de la economía de mercado puede existir lo que Mises denominó como intervencionismo (Interventionism). En este tipo de sistema, se respeta la propiedad privada y el mercado, pero el gobierno interviene en su funcionamiento. La conducta intervencionista busca incidir sobre el aparato productivo por medio de órdenes y prohibiciones ${ }^{8}$, aplicadas, en muchos casos, de manera coercitiva. Según Mises: "Se busca preservar el dualismo entre la esfera de las actividades gubernamentales de una parte y la libertad económica bajo el sistema de mercado por la otra" (1996[1949]: 698).

Sin embargo, Mises muestra cómo el monopolio no es patrimonio exclusivo del sistema capitalista. Tanto el socialismo (en el modelo ruso como en el alemán) y en el intervencionismo el monopolio surge como la herramienta para beneficiar a la sociedad, bajo el lema "La ganancia del monopolio son los fondos que generan la ganancia social" (Mises 1996[1949]:407408). Para Mises, un fenómeno de la economía y la administración contemporánea es que el gobierno promueve el intervencionismo y los monopolios y apunta que el problema no es la correcta delimitación de lo "natural", lo "justa" o "apropiadas" tareas del Estado o el gobierno, sino ¿Cómo funciona un sistema de intervencionismo? (1996[1949]: 703). Según Mises, el sistema funciona de acuerdo a lo que el gobierno considera que es "socialmente deseable" (1996[1949]: 704). Esto significa, según Mises:

"Que el mercado es libre si hace precisamente lo que el gobierno desea hace. Esto es "libre" de hacer lo que las autoridades consideran que es "bueno", pero

7 Según Mises: "What these neosocialist suggest is really paradoxical. They want to abolish private control of means of production, market exchange, market prices, and competition. But at the same time they want to organize the socialist utopia in such a way that people could act as if these things were still present. They want people to play market as children play war, railroad, or school. They do not comprehend how such childish differs from the real thing it tries to imitate" (1996[1949]: 686).

8 Alguna de las estrategias por medio de los cuales la burocracia gubernamental controla a las empresas son: 1) influencias en la selección de juntas directivas de, refiriéndose el Reino Unido (Mises, 1983[1944]: 77); 2) medidas discrecionales adoptadas por organismos públicos de promoción o control (Mises, 1983[1944]: 78); 3) el control del mercado de divisas (Mises: 1983[1944]: 79); o 4) el uso de la diplomacia o el soborno (Mises, 1983[1944]: 79). 
no lo que ellos consideran que es "malo"; la decisión acerca de lo bueno y lo malo descansa en el gobierno. Así la doctrina y la práctica del intervencionismo finalmente tiende a abandonar lo que originalmente lo distingue del socialismo y adopta completamente los principios de la planificación totalitaria" (1996[1949]: 704).

\section{Propuestas de Mises expuestas en Omnipotent Government}

En el capítulo III denominado Estatism, Mises observa que el principal acontecimiento de las últimas décadas fue el desplazamiento del liberalismo por el estatismo. Al igual que en Human Action, Mises considera que el estatismo aparece en dos formas: socialismo e intervencionismo. Sin embargo, el socialismo y el intervencionismo comparten un mismo fin: subordinar al individuo incondicionalmente al Estado, el aparato social de compulsión y coerción" (Mises, 1944: 54). Para Mises, la frase que soporta mejor el discurso del estatismo fue lo que $\mathrm{Hegel}^{9}$ expuso en su Filosofía del Derecho cuando dijo que el Estado es absolutamente racional y que posee autoconsciencia. Esas propiedades le permiten, dice Mises, guían y tutelan al ciudadano, restrin- giendo su libertad de actuar, moldeando su destino.

Para Mises el Estado es un aparato de compulsión, pero no todo aparato de compulsión se puede llamar Estado. Para adquirir el derecho de existir, un Estado, aparte de contar con población, territorio, se requiere contar con el suficiente poder político para mantener su existencia (soberanía) al menos por un tiempo más o menos prudencial sin interferencia de otras fuerzas exógenas a ese poder. El total de reglas que garantizan el poder son las leyes y son aplicadas por el gobierno como representación del Estado.

Ahora bien, para Mises la característica esencial del Estado y el gobierno no depende de su estructura y constitución particular, sino porque son sistemas en donde pueden existir mayorías y minorías. Para Mises, las mayorías no son necesariamente buenas para un Estado, ya que las mayorías no están menos expuestas a los errores y desastres como los dictadores o reyes.

El sistema socialista es, para Mises, una de las formas más acabadas de estatismo surgidas en el siglo XX. Considera que hablar de Estado socialista es un pleonasmo, ya que socialismo necesariamente implica propiedad gubernamental de los medios de producción de la sociedad. Esto es la existencia de empre-

9 Para Mises, hubo también, obviamente otros intelectuales que contribuyeron a difundir las ideas de estatismo tales como Simone de Sismondi, Robert Owen, Saint Simon, Auguste Compte, Lorenz von Stein, Frederick List, Alexander Hamilton, Karl Marx o Friedrich Engels, entre otros. Además agrega que los estudiantes de aquella época se les enseñó a admirar el "Reino social" de los Hohenzollerns, el "Noble socialista" Federico Guillermo I, a Guillermo I, el "Gran Kaiser de la seguridad social y la legislación laboral". 
sas del Estado en casi todo tipo de negocio, se substituye al empresario por el burócrata o el Betriebsführer. De hecho, Mises apunta que el gerente en una economía socialista no tiene necesidad de ajustar las operaciones de la empresa o Sovjos, Kombinat o, como dice Voslensky (1981), el "Ultra-monopolio" a los requerimientos del mercado ${ }^{10}$, ya que de hecho funcionan como monopolistas de oferta o monopolistas de demanda (monopsonio). La toma de decisiones empresariales (de mercadeo, ventas, producción o financieras, entre otras áreas) no están sujetas a los cambios previstos o no en la conducta de los consumidores. La empresa estatal, según Mises, es la autoridad de conceder favores como hermano mayor o padre de familia. En el sistema socialista las empresas del estado encuentra un fuerte dificultad, dice Mises, de someterse al control parlamentario, debido a que su presupuesto público debe ser aprobado en una instancia también pública que forma parte del Estado. La solución que se ha encontrado es establecer un sistema de planificación centralizada que, en un régimen de partico único, bajo el concepto de "centralismo democrático" obvia, ignora o permite la aprobación de presupuestos que posteriormente no soportarían los cuestionamientos de un sistema parlamentario de partidos diferentes al del gobierno. Por esta razón Mises dice: "Thus socialism must lead to the dissolution of Democracy. The sovereignty of the consumers and the democracy of the market are the characteristic feature of the capitalism system" (Mises, 1944: 63). Estas fuertes debilidades, que hacen ser al socialismo un sistema débil desde el punto de vista administrativo se deriva, como lo expresó Mises en Human Action, por la imposibilidad de aplicar el cálculo económico. Sin embargo, aquí Mises precisa más aún porqué esa falla. En un sistema en que todos los factores de producción son propiedad del Estado y el mercado (por medio de la función de los precios como mecanismo de asignación de recursos) los gerentes socialistas se encuentran con las siguientes dificultades: 1) no pueden saber si aquello que están planeando y ejecutando es razonable desde el punto de vista económico; 2) no cuenta con medios para discernir acerca de qué medios y métodos de producción bajo consideración son los más adecuados; 3 ) no se puede hallar una genuina base de comparación entre las cantidades de los diferentes insumos, servicios y factores de producción; 4) con lo anterior, el gerente socialista no puede comparar los desembolsos necesarios con los resultados anticipados, ya que eso sólo lo pueden ofre-

10 En esta cita que Michael Voslensky tomo de Kommunist, se expone el papel de los administradores y los trabajadores en la economía socialista: "La disciplina del trabajo socialista comporta, por un lado, la obligación, de parte de los administradores, de organizar racionalmente el trabajo; por otro lado la obligación por parte de los obreros y los empleados, de consagrar todas sus fuerzas al trabajo [...]. En cuanto a la organización racional del trabajo es: La aptitud para organizar el trabajo exige la utilización máxima de las horas de trabajo, la creación de una atmosfera al trabajo, la contabilidad y el control" (1981: 156). 
cer los precios de referencia del mercado para los productos directamente involucrados en el trabajo a realizar.

En conclusión, prevalece el criterio de que es más importante culminar la obra, tarea, actividad o lo que sea sin importar el costo asumido. Es por esta razón que según, Mises, se impone un sistema de burocrático al estilo ruso ${ }^{11} \mathrm{o}$ el sistema alemán de Zwangswirtschaft (economía de la coacción).

Es decir, impera más la lógica del ingeniero o de los medios en si mismo que la postura equilibrada (en términos de necesidades y recursos escasos) del economista en términos de Robinson (1980[1932]). Por otra parte, en ocasiones parece ser que los sistemas como los descritos por Mises obvian el papel de la escases de recursos en relación a los fines. En primer lugar, para Robbins: "La condición de escases de los bienes no es "absoluta". La escasez no significa una mera falta de frecuencia, sino limitación con respecto a la demanda" (1980 [1944]: 74). En segundo lugar, independientemente de las críticas que se puedan levantar en contra de Robbins: "El mundo externo no ofrece oportunidades cabales para su logro completo. La vida es corta. La naturaleza es mezquina. Nuestros semejantes tienen otros propósitos..." (1980 1944: 35). En consecuencia, las observaciones de Mises apuntan al intento de convertir a la Economía concebida: "Como parte de la Ética o la Estética, es decir, como disciplinas que estudian los fines por sí mismos" (Robbins, 1980 [1944]: 57).

\section{Conclusiones}

En las tres obras antes mencionadas, el discurso administrativo de Ludwig von Mises se concentra en los siguientes aspectos: 1) la burocracia existe tanto en el sistema económico capitalista como en el sistema económico socialista; 2) sin

11 Según Voslensky (1981) Vladimir I. Lenin así como Levenstern, Poliakov, Trotsky, Pankin y especialmente Alekséi Gastev (quien introdujo los métodos de trabajo y entrenamiento de obreros basados en procesos psicológicos y biológicos en el trabajo) eran partidarios de la Organización Científica del Trabajo o Nauchnaia organizatsia truda (NOT) o en otras palabras de los principios Frederick Winslow Taylor. Para tal fin, el Consejo Central de Sindicatos creó en 1920 el Instituto Central del Trabajo (ICT). En 1921 se reunió la primera conferencia panrusa sobre los problemas de la OCT. Para 1926 existían 60 ICT, lo que obligó la creación de un Consejo Central de la Organización Científica del trabajo. Junto a este conjunto de instituciones, Lenin propuso en diciembre de 1917 la "organización de la emulación socialista del trabajo" y en abril del mismo año surgió la llamada gran iniciativa conocida como el "Subbótniki/Voskrésniki" (días de trabajo voluntario sin remuneración salarial), el movimiento stajanovista (movimiento que comenzó en 1935 como forma de generar la emulación o competencia dentro del socialismo o sotsialsticheskoie sovevnovanie con aumento de la productividad de los trabajadores por su iniciativa. Su nombre proviene del minero del Donetsk Alekséi Stajánov. Este movimiento supuso la eliminación del koljoz en el sector industrial), las brigadas de trabajo comunista, el movimiento de trabajadores de choque (Udarniki), la creación de brigadas del Konsomol (Unión de Jóvenes Comunistas, fundada en 1918 y agrupaba jóvenes entre 14 y 28 años de edad y eran un mecanismo para propagar las ideas del socialismo). 
embargo, la burocracia en el totalitarismo es diferente que en el sistema democrático; 3) el estatismo surge en el socialismo bajo la forma de dos variantes: la variante rusa y la variante germana; 4) el estatismo también aparece en el capitalismo bajo la forma de intervencionismo, al estilo, por ejemplo, del New Deal en los Estados Unidos o la Socialpolitik en la República de Weimar; 5) tanto el sistema burocrático público como el de la empresa se sustentan en presupuestos; 6 ) en el sistema burocrático público los presupuestos son blandos, mientras que en el sector privado son rígidos; 7) en consecuencia, ante déficits públicos, los gobiernos hacen uso de los impuestos o el endeudamiento, mientras que en el sector privado se debe hacer ajustes.

En las tres obras expuestas Mises encuentra que el sistema administrativo socialista debe enfrentar una serie de problemas surgidos de su propia doctrina de "superar al sistema capitalista", por medio de la eliminación de la propiedad privada y su substitución por la propiedad social (estatal). Entre estas serias dificultades se encuentran: 1) que no se ha resuelto el problema de la transformación; 2) el no empleo del cálculo económico; en consecuencia, el ideal de la planificación socialista centralizada naufraga, debido a la inexistencia de un referente claro en términos de precios para tomar decisiones administrativas acertadas; 3 ) el gerente socialista confunde la habilidad que puede eventualmente tener una persona para predecir el futuro con la tarea de diseñar realizar el cálculo económico bajo la forma de presupuestos, planes o programas; 4) peor aún, el gerente socialista debe enfrentarse a la imposibilidad de an- ticipar y actuar en consecuencia ante las expectativas a corto plazo; 5) la cuestión se complica cuando el gerente socialista debe abordar la toma de decisiones a largo plazo, que implica las expectativas a largo plazo, que son más inestables precisamente por que el gerente debe contar con su ignorancia con respecto al futuro, la inseguridad de lo que cree saber acerca del futuro y la incertidumbre que afecta determinado negocio, sector económico o la economía en su conjunto; 6 ) en consecuencia, el gerente en el sistema socialista también se encontrará con el hecho de que habiendo oportunidades de inversión, la falta de capacidad para determinar cuál es la más conveniente no necesariamente lo capacitaría para promover el inhibirse o evitar el tener que seleccionar determinada alternativa; 7) dado lo antes expuesto, el gerente socialista solamente se tendrá que limitar a cumplir un plan decidido en un escenario extraeconómico, debe avocarse a implementar la decisión previamente tomada, que en el trayecto puede haber sido la peor posible; 8) en otras palabras, no se trata de tomar decisiones óptimas, que sería lo mejor, sino en tomar buenas decisiones económicas con recursos escasos y fines ilimitados; 9) la anterior circunstancia lleva a que el gerente socialista debe suponer de que siempre el Estado contará con reservas de materias primas o tierras en proporción casi infinitas como para pagar los costo incurridos y los errores cometidos (Schumpeter, 1994b [1942]); 10) por otra parte, el gerente socialista debe portarse más como ingeniero que como administrador/economista, debido a que hay una separación entre los fines, decididos en el plan (ex-ante, o por lo menos de difícil 
cambio, debido al proceso burocrático que implica revertir todo o parte del proyecto) se debe afrontar para, y los medios o lo que debe emplear para cumplir con lo establecido en el plan, también decididos de antemano.

Lo antes expuesto, supone que el gerente socialista, al afrontar, en términos generales, las mismas disyuntivas que un gerente en la economía capitalista, se encuentra con menos herramientas para minimizar los efectos negativos de las decisiones surgidas en la planificación central. Esta limitación se encuentra en: la carencia de la herramienta del cálculo económico basado en el mercado; la separación entre fines y medios, ya que los fines son éticos en sí, pero requieren tener como referente a los medios disponibles escasos; $y$ la imposibilidad de ajustar sus expectativas a los cambios del entorno económico, debido a que no hay mercado o si existe no sirve de referente.

En consecuencia, si bien es cierto que se busca el bienestar del hombre, este no puede ser a cualquier costo e ignorando que existen recursos limitados. La circunstancia antes mencionada llevó a Kornai (1979) a exponer su tesis de insuficiencia de la producción y del ajuste macroeconómico por vía de los precios. Más aún, este sistema administrativo se presta a adquirir mucho de los vicios que aquejan al sistema administrativo capitalista tales como: los efectos de agencia; concentrar los beneficios y esparcir pequeñas cuantía de los costos sociales en un gran número de personas, debido a la existencia de los intereses especiales (Friedman, 1993); o la tendencia, debido a razones ideológicas o de intereses es- peciales a crear grandes monopolios o supermonopolios estatales.

\section{Referencias bibliográficas}

AC-URSS (Academia de Ciencias de la Unión Soviética) (1975). Manual de economía política. Barcelona: ediciones Grijalbo, S. A.

Barone, Enrico (1908). II Ministero della produzione nello Stato Collettivista. Milano: Giornali degli Economisti, sep/oct., 2, pp. 267-293, 392-414 (Università Bocconi).

Bergson, Abram (1967). Market socialism Revisited. The Journal of Political Economy. 75(5), pp. 655-673 (University of Chicago).

Dietrich, Heinz Stefan (2007). El socialismo del siglo XXI. Primera reimpresión. Bogotá: Fundación para la Investigación y la Cultura.

Ekelund, Robert y Hébert, Robert (1992). Historia de la Teoría Económica y de su Método. Tercera edición. McGraw Hill/interamericana de España, S.A. Madrid.

Friedman, Milton (1993). Why Government is the problem. Essay in public policy. Stanford: Hoover Institution, Stanford University.

Gregory, Paul y Stuart C. Robert (1989). Comparative Economic System. Third edition. Boston: Houghton Mifflin Company.

Kornal, Janos (1979). Resource-constrained versus Demand-constrained Systems. Econometrica Vol. 47, pp. 801-819 (The Econometric Society, Chicago).

Lange, Oskar (1976) Problemas de economía política del socialismo. Segunda reimpresión. Bogotá: Fondo de Cultura Económica. 
Lenin, Vladimir I. (2007[1917]). El Estado y la Revolución. Buenos Aires: Longseller.

Marx, Karl (1976 [1867] a). El capital. Crítica de la economía política. Tomo I. Duodécima reimpresión. Bogotá: Fondo de Cultura Económica.

Marx, Karl (2008 [1857-61]). Grundrisse der Kritik der Politischen Ökonomie. Source Penguin 1973. Traducido por Martin Nicolaus. [En línea fecha de documento 2002] [http://www.marxists.org/archive/marx/works/1857/ grundrisse/] [Consulta A 08-03-2008].

Mises, Ludwig Elder (1983[1944]). Bureaucracy. Grove City, PA: Libertarian press, Inc.

Mises, Ludwig Elder (1983[1944]). Omnipotent Government. The Rise of the Total State and Total War. New Haven: Yale University Press. Pp. 301.

Mises, Ludwig Elder (1996[1949]) Human Action: A Treatise on Economics. $4^{\text {th }}$ revised edition. Forward by Bettina Bien Braves. Irvington on Hudson, NY: The Foundation for Economic Education. Pp. 920. Disponible en lengua inglesa en el site web http// www.econlib.com.

Movimiento V República (1996). Agenda Alternativa Bolivariana. Una propuesta patriótica para salir del laberinto. Caracas: Proyecto Nacional V República.

Nash, George H (1987[1976]). La Rebelión Conservadora en Estado Unidos. Buenos Aires: Grupo Editor Latinoamericano, S. R. L. edición en inglés: "The Conservative Intellectual Move- ment in America since 1945". New York: Basic books Publishers.

Robbins, Lionel (1980[1944]). Ensayo sobre la Naturaleza y Significación de la Ciencia Económica. México, D. F.: Fondo de Cultura Económica. Pp. 208.

Schumpeter, A. Joseph (1994a) Historia del análisis económico. Barcelona: Ariel Economía.

Schumpeter, A. Joseph (1994b [1942]). Capitalism, Socialism and Democracy. London: Routledge Taylor and Francis Group.

Vanek, Jaroslav (1970). The General Theory of Labor-Managed Market Economies. New York: Cornell University Press.

Voslensky, Michael (1981). La Nomenklatura. Los privilegiados en la URSS. Barcelona: Argos-Vergara.

Ward, Benjamin (1958). The firm of Illyria: Market Syndicalism. American Economic Review, 48 (September), pp. 566-589.

Weber, Max (1977[1922]) Economía y Sociedad: Esbozo de una Sociología comprensiva. Tomo I. Tercera reimpresión. México, D. F.: Fondo de Cultura Económica. Título original en alemán: "Wirtschaft un Gesellschaft Grudriss der Verstehenden Soziologie".

Zambrano, María Luisa (2008). Desarrollo endógeno socialista y defensa popular. Una propuesta a partir de los Consejos Comunales. Caracas: Liven Editores, S. A. 